\title{
Clinical Profile and Treatment Outcome of Obstructive Uropathy in a Tertiary Care Centre
}

\author{
${ }^{1}$ Dilip Kumar Pal, M.S., M.Ch, ${ }^{2}$ Surajit Sasmal, M.S \\ ${ }^{1}$ Professor \& Head, Department of Urology, \\ Institute of Post Graduate Medical Education \& Research, India \\ ${ }^{2}$ Post Doctoral Trainee, Department of Urology, \\ Institute of Post Graduate Medical Education \& Research, India
}

\begin{abstract}
Introduction: Obstructive uropathy is one of the most common urological emergencies. It can lead to irreversible kidney damage. The etiology is largely determined by the patient's age. It can be due to benign or malignant causes. This study aimed at determining the profile and outcome of patients with obstructive uropathy.

Methods: A Prospective observational study carried out including patients with a diagnosis of obstructive uropathy. Following admission all patients of the study population underwent complete urine analysis, urine culture and sensitivity, serum creatinine, and complete Blood count, followed by ultrasonography. Abdominal ultrasonography (US) was carried out in all patients. Following Double J stenting(DJ stenting), percutaneous nephrostomy $(P C N)$, per urethral catheteriasation $(P U C)$ or suprapubic cystostomy $(S P C)$, serum creatinine, urine output, serum electrolyte(sodium, potassium) was recorded on dayl, day3, day7, and day 28 \& at the end of $3 \mathrm{rd}$ month .In our study, serum creatinine level $<1.5 \mathrm{mg} / \mathrm{dl}$ and complete weaning from dialysis was taken as recoverable group
\end{abstract}

Results: Out of 139 patients $64.7 \%$ were males. The mean age was 45.7 years. $38.85 \%$ patients needed emergency dialysis. Symptoms at presentation: loin pain $64.74 \%$ followed by vomiting $46.04 \%$, oliguria $17.08 \%$ \& urinary retention $12.23 \%$. Urinary tract infection was present in 15 patients. Main etiologies of obstruction were urolithiasis (35\%) and benign prostatic hypertrophy (20.1\%). The most common cause of malignancy related obstructive uropathy was carcinoma cervix (50\%) with carcinoma urinary bladder being the second most common cause (20\%).

96.4\% patients underwent different modes of drainage while $3.6 \%$ needed direct operation. $87 \%$ patients recovered completely. Presence of malignancy, infection \& increased time interval between disease onset \& intervention affected the renal recoverability significantly having $p$ value of 0.037, 0.042 and 0.001 respectively. However presence of anaemia lost its statistical significance in affecting the renal recoverability.

Conclusion: Obstructive uropathy can affect any age group. It has got no sex predilection. Symptoms can be variable with pain being the most common symptom. Urinary stones and BPH are the common benign causes while cervical cancer and bladder cancer account for the majority of malignancy related obstructive uropathy. In most cases renal recovery is good. But presence of malignancy, infection \& increased time interval between disease onset \& intervention affected the renal recoverability significantly.

Keywords: Obstructive uropathy, haemodyalysis, serum creatinine

\section{INTRODUCTION}

Obstructive uropathy refers to the structural or functional changes in the urinary tract that impede normal urine flow. Obstructive uropathy is one of the most common urological emergencies with an overall incidence of $20 \%(1)$.

It may be acute or chronic, complete or incomplete, unilateral or bilateral and can lead to rapid deterioration in renal function and irreversible kidney damage if urinary drainage is not rapidly Corrected $(2,3)$.

The etiologies are diverse, can be benign or malignant, largely determined by the age of the patient. In children the main aetiologies are uretero-pelvic junction obstruction and congenital urethral valves 
and meatal stenosis (4-6). In young adults, calculi are primary cause while in older patients benign prostatic hyperplasia, calculi and malignancy are the common causes (6).

The signs of obstructive nephropathy are often nonspecific and variable depending on the time interval over which the obstruction occurred as well as the lateralization and the severity of obstruction. The pattern of clinical presentation can be loin pain, lower urinary tract symptoms, fever, mass effect, urine retention, and anuria, impaired renal function with uremic signs $(6,7)$.

Regardless of the patient's age, appropriate diagnosis and prompt surgical or interventional drainage is necessary to avoid irreversible renal damage (8). It is often-reversible and the degree of renal recovery depends primarily on the extent and duration of the obstruction together with the presence or absence of other co morbidity (9).

A large gap of knowledge exists about the clinical profile and treatment outcome of the patients with obstructive uropathy which needs to be bridged by various systematic studies in future.

The present study has been performed in a prospective manner to evaluate influence of clinical profile of the patient, time interval between appearance of symptoms and intervention taken, presence of infection, malignant versus benign cause of obstruction on renal recoverability.

\section{Materials AND MethodS}

This study has been done after obtaining approval from Institutional Ethical Committee ( Memo No.Inst/IEC/2015/196) This is a prospective study done from March 2015 to November 2016.During this period a total of 139 patients with obstructive uropathy due to stone, stricture, PUJO; malignancy like prostate, bladder, cervix were included. Those patients who developed hematuria after catheterization were admitted and included in the study. Patient with urethral stricture disease underwent per urethral or suprapubic catheterization on outpatient department basis and were not included in the study. Patients known to have chronic kidney disease, Patient having previous percutaneous nephrostomy drainage (PCN drainage) or double J stenting (DJ stenting) were excluded from this study. Patients underwent complete urine analysis, urine culture and sensitivity, blood chemistry: Serum creatinine, Serum sodium (Na), Serum potassium (K), followed by Plain X-ray KUB, abdominal ultrasonography, non contrast computer tomography (non contrast CT scan) (in selected cases).We have done haemoglobin level estimation once at the time of admission. Initially we provided temporary relief measures like DJ stenting or PCN drainage and dialysis was given as per requirement. Definitive treatment was done later. All patients were put under strict clinical surveillance during the early postoperative days. The total duration of follow up ranged from two weeks to six months after definitive procedures. On post operative day 1 and post operative day 3 following investigations and parameters were evaluated -Urine output, urine analysis with culture and sensitivity test, serum creatinine, serum urea, serum electrolytes $(\mathrm{K} \& \mathrm{Na}$ ). At the end of 1 weeks following investigations were done - monitoring of urine analysis with culture and sensitivity test, serum creatinine, serum urea, serum electrolytes $(\mathrm{K} \& \mathrm{Na})$ level will be done. Abdominal ultrasonography (USG) was performed after 2 week. The need of re-dialysis was assessed. Then patient were reviewed 2 weekly gap for 3 months with previously mentioned biochemical reports. Evidence of total recovery was judged by one or more of the following criteria

- Creatinine retuning to the normal level.

- Complete weaning from dialysis occurred.

Partial recovery was defined by one or more of the following criteria

- Creatinine decreased but non achievement of the normal level.

- Decrement of number of weekly dialysis sessions.

Patients having no evidence of complete or partial recovery were considered having no recovery

\section{Statistical Analysis}

Continuous data was presented as mean +/- standard deviation. Categorical data was expressed in percentage. Chi-square test can be used to determine significant differences between two groups. Here two groups are made according to patient recovery status (total recovery vs. partial and no recovery). Odds ratio was determined as required. Significance of the test was determined by probability value of less than $.05(\mathrm{p}<.05)$. 


\section{RESULTS}

The commonest symptom was loin pain $64.74 \%$ followed by vomiting $46.04 \%$, oliguria $17.08 \%$ \& urinary retention $12.23 \%$ (Table1). Commonest etiology was urolithiasis (32.4\%), followed by prostatic hypertrophy $(20.1 \%) \&$ stricture $19.4 \%$ (Table 2). Amongst the malignancy types cervical cancer accounted the most $50 \%$ followed by bladder tumour $20 \%$, cancer prostate $15 \%$. $96.4 \%$ population underwent different modes of drainage while $3.6 \%$ needed direct operation. Maximum were treated by DJ Stenting $42.45 \%$, followed by PCN 31.65\%, PUC 19.5\%, and SPC 3\% (Table 3). $38.85 \%$ Population underwent urgent haemodialysis (Table 4). $87 \%$ population recovered fully. Presence of malignancy, presence of infection \& increased time interval between disease onset \& intervention affected the renal recoverability significantly having $\mathrm{p}$ value $0.037,0.042,0.001$ respectively as shown in (Table 5,6$)$. However presence of anaemia lost its statistical significance in affecting the renal recoverability $\mathrm{p}$ value 0.944 .

Table1. Clinical profile of the patient $(n=139) *$

\begin{tabular}{|c|c|c|}
\hline Symptoms & Total population & Percentage \\
\hline Loin Pain & 90 & $64.74 \%$ \\
\hline Vomitting & 69 & $46.04 \%$ \\
\hline Fever & 10 & $7.19 \%$ \\
\hline Limb Oedema & 10 & $7.19 \%$ \\
\hline Anuria & 11 & $7.91 \%$ \\
\hline Oliguria & 25 & $17.98 \%$ \\
\hline Resp Distress & 7 & $5.03 \%$ \\
\hline AcuteUrinary Retention & 17 & $12.23 \%$ \\
\hline Lower Urinary tract Symptoms & 15 & $10.79 \%$ \\
\hline
\end{tabular}

* Multiple responses

Table2. Distribution of total population according to etiology $(n=139)$

\begin{tabular}{|c|c|c|}
\hline Etiology & Total population & Percentage \\
\hline Stone & 45 & 32.4 \\
\hline BPH & 28 & 20.1 \\
\hline Stricture & 27 & 19.4 \\
\hline Malignancy & 20 & 14.4 \\
\hline Others** & 19 & 13.7 \\
\hline Total & 139 & 100 \\
\hline
\end{tabular}

** Others include retro peritoneal fibrosis, retro peritoneal benign tumor etc.

Table3. Distribution of population according to treatment provided $(n=139)$

\begin{tabular}{|c|c|c|}
\hline Treatment Modalities & Population & Percentage \\
\hline Dj Stenting & 59 & 42.45 \\
\hline Pcn & 44 & 31.65 \\
\hline Direct Ot & 5 & 3.6 \\
\hline Puc & 27 & 19.5 \\
\hline Spc & 4 & 3 \\
\hline Total & 139 & 100 \\
\hline
\end{tabular}

Table4. Distribution of population according to Hemodialysis requirement $(n=139)$

\begin{tabular}{|c|c|c|c|}
\hline \multicolumn{2}{|c|}{ HD needed } & Population & Percentage \\
\hline \multirow{3}{*}{ YES (N=54) } & Refractory Hyperkalemia & 30 & 21.58 \\
\cline { 2 - 4 } & Uremic Symptoms & 14 & 10.07 \\
\cline { 2 - 4 } & Uro Sepsis & 10 & 7.2 \\
\hline \multicolumn{2}{|c|}{ NO(N=85) } & 85 & 61.15 \\
\hline \multicolumn{2}{|c|}{ TOTAL } & 139 & 100 \\
\hline
\end{tabular}

Table5. Correlation between infection \& recovery $(n=139)$

\begin{tabular}{|c|c|c|c|}
\hline Infection & Total Recovery & Partial +No Recovery & Odds Ratio(P Value) \\
\hline Present & $12(7 \%)$ & $3(30 \%)$ & $0.85(0.042)$ \\
\hline Absent & $117(93 \%)$ & $7(70 \%)$ & \\
\hline Total & 129 & 10 & \\
\hline
\end{tabular}


Dilip Kumar Pal \& Surajit Sasmal

Table6. Correlation between time interval \& recovery $(n=139)$

\begin{tabular}{|c|c|c|c|}
\hline TIME INTERVAL $* * *$ & TOTAL RECOVERY & $\begin{array}{c}\text { PARTIAL +NO } \\
\text { RECOVERY }\end{array}$ & Odds Rati (P Value) \\
\hline >20 Days & $39(30 \%)$ & $8(20 \%)$ & \multirow{2}{*}{$0.1(0.001)$} \\
\hline <20 Days & $90(70 \%)$ & $2(80 \%)$ & \\
\hline TOTAL & 129 & 10 & \\
\hline
\end{tabular}

*** Time interval means time gap between disease onset and intervention (DJ stenting, PCN, catheterization or Haemodialysis) taken.

Table7. Correlation between anaemia \& recovery

\begin{tabular}{|l|l|l|l|l|l|}
\hline & & Total Recovery & Partial/No Recovery & Total & P value(chi squaretest) \\
\hline HEMOGLOBIN & $<10 \mathrm{gm} / \mathrm{dl}$ & 27 & 2 & 29 & 0.944 \\
\cline { 2 - 5 } & $>10 \mathrm{gm} / \mathrm{dl}$ & 102 & 8 & 110 & \\
\hline \multirow{2}{*}{ Total } & 129 & 10 & 139 & \\
\hline
\end{tabular}

Table 8A. Serum creatinine at different follow up days

\begin{tabular}{|c|c|c|}
\hline Creatinine level & Mean(mg/dl) & Standard deviation \\
\hline D0 & 4.6 & 3.3 \\
\hline D1 & 2.8 & 1.9 \\
\hline D3 & 2.0 & 1.1 \\
\hline D7 & 1.6 & 0.7 \\
\hline D28 & 1.45 & 0.5 \\
\hline M3 & 1.3 & 0.3 \\
\hline
\end{tabular}

Table 8B. Serum BUN at different follow up days

\begin{tabular}{|c|c|c|}
\hline BUN level & Mean(mg/dl) & Standard deviation \\
\hline D0 & 50.98 & 33.57 \\
\hline D1 & 34.58 & 23.56 \\
\hline D3 & 23.71 & 15.23 \\
\hline D7 & 22.14 & 14.24 \\
\hline D28 & 21.23 & 13.62 \\
\hline M3 & 22.19 & 14.26 \\
\hline
\end{tabular}

Table 8C. Serum potassium at different follow up days

\begin{tabular}{|c|c|c|}
\hline K+ level & Mean(meq/dl) & Standard deviation \\
\hline D0 & 4.2 & 0.9 \\
\hline D1 & 3.9 & 0.6 \\
\hline D3 & 3.8 & 0.4 \\
\hline D7 & 3.7 & 0.3 \\
\hline D28 & 3.6 & 0.4 \\
\hline M3 & 3.7 & 0.3 \\
\hline
\end{tabular}

D0-Just after admission,D1-On Day1, D2- On Day 2,D3-On Day 3, D7-On Day7, D28-On Day 28

M3- After Month 3

\section{DISCUSSION}

Obstructive uropathy is a common urological emergency which results due to obstruction of urine flow resulting in increased pressure of the tract; if not treated in time can result in renal parenchymal damage leading to renal failure. In this study various etiologies of obstruction, clinical profile of patient, and different modes of relieve obstruction with regards to time of intervention and outcome in obstructive uropathy in patients attending department of Urology.

In this study maximum population belonged to the age group of 50-59 years with mean age being 45.7 years. The eldest being 73 years old \& youngest being 4 years old .

Gender wise males $(64.7 \%)$ outnumbered females $(35.3 \%)$ having male: female ratio of 1.857:1 
Our study revealed urolithiasis being major cause for obstruction accounting $32.4 \%$ followed by, benign prostatic hypertrophy \& stricture. Those who suffered from malignancy mainly of cervical and bladder region accounted the most. BPH is a problem experienced by aging men and is the most common benign aetiology of obstruction in men, our result are similar to the literature were BPH account for $30 \%$ of obstruction in one series. Hydronephrosis is a common situation in cases of advanced malignancies, and the cause of obstruction may be invasion of the ureters by tumor, extrinsic compression by a retroperitoneal primary or metastatic neoplasia. Cancer was the cause of obstruction in $14.4 \%$ in our participants. This rate is high compare to the study of EI Iman (4) in Soudan where cancer accounted only for $8 \%$ of cases. The difference could be due to the fact that our study was done in a tertiary referral hospital where patients with malignancy in the region are usually referred late.

Signs of obstructive uropathy are often non-specific, depending on the time interval over which the obstruction occurs, the lateralization and severity of obstruction. Although a decrease in urine output frequently observed, normal or elevated urine output does not exclude partial obstruction. In the present study most patients presented with loin pain (64.7\%), vomiting (49.7\%), oliguria (18\%) \&acute urinary retention (12.2\%) Patients presented with various symptoms especially uremic sign, sign of volume over lode and loin pain. The explanation is the late presentation of patients with severe renal function deterioration and also by the etiology of the obstruction that was mainly urolithiasis and cancer in this study. These findings are consistent with others studies such as in 2005 Rishi Nayyar et al.(10) who also showed that urinary obstruction is a common cause of acute and chronic renal failure. The symptoms and signs of an obstructed kidney may vary from asymptomatic to severe acute pain.

Drainage was done in $96.4 \%$ of patients, DJ stenting being $42.45 \%$, PCN being 31.65 , rest being catheterization. Only $3.6 \%$ needed direct operation.

Urinary tract obstruction is a common clinical problem facing urologist. It may be acute or chronic, partial or complete, unilateral or bilateral, can occur at any site of the urinary tract and lead to rapid deterioration in renal function and irreversible kidney damage if urinary damage is not corrected in a time. Once the diagnosis of obstructive uropathy is made, prompt and appropriate intervention is necessary to avoid irreversible renal damage. Active surgical intervention and creation of adequate urine outflow from the obstructed kidney is the method of choice for initial treatment even in case of malignancy irrespective of the disease stage. The results in our study are consistent with the literature: Obstruction is a relatively common cause of kidney failure but the rate of severe renal insufficient with a need for emergency dialysis in our study is comparable (38.8\%) to a similar study buy Iman in Sudan where $40 \%$ presented with significant renal impairment and required emergency dialysis.

Certain patient-specific factors especially CKD traditional risk factors may increase the risk of kidney function deterioration amongst patients with obstruction. The degree of renal recovery depends first on the extent and duration of the obstruction together with the presence or absence of infection. Total renal recovery occurred in $87 \%$ of cases in this study and was partial in $9 \%$. This is comparable to the study done by Singh et al.(11) in 1982 who reported renal function improvement in 86\%, while 6\% showed no improvement and they were given regular dialysis after surgical management of 50 patients with renal and ureteric calculi and renal failure ( 31 acute renal failures and 19 chronic renal failures).

Based on our observations, patients with bilateral obstruction secondary to malignant cancer should be counseled that their prognosis is poor. These results are consistent with other reports in which a malignant cause of obstructive uropathy is considered as a prognostic indicator of morbidity and reduced survival.

Poor recovery has been associated with presence of infection, prolonged time interval ( $>20$ days) between disease onset $\&$ recovery $\&$ those who suffered from malignancy having p value $0.042,0.001$ $\& 0.037$ respectively. Co morbidities, age, sex \& presence of anaemia has lost statistical significance in affecting the recovery. 


\section{Conclusion}

Obstructive uropathy is one of the major cause of morbidity patients attending urology outpatient department. It can affect anybody ranging from a toddler to geriatric population with increased occurrence in advanced age. It has got no sex predeliction. Symptoms can be varied: pain being the most common. Urinary stones and BPH are the common benign causes while cervical and bladder cancer account for the majority of malignancy. In most cases renal recovery is good. Malignancy being associated with poor recovery, patient should be counseled before going for intervention. Infection of urinary tract should be aggressively handled as it is associated with increased morbidity

\section{REFERENCES}

[1] Sood G, Sood A, Jindal A, Verma DK, Dhiman DS. Ultrasound guided percutaneous nephrostomy for obstructive uropathy in benign and malignant diseases. Intl Braz J Urol.2006; 32:281-86.

[2] Ishidoya S, Kaneto H, Fukuzaki A, Takeda A, Ogata Y,Nakagawa H, Orikasa S, Arai Y. Pathophysiology and clinical implication of obstructive nephropathy.NihonHinyokika GakkaiZasshi.2003; 94:645-55.

[3] Tang X, Lieske JC. Acute and chronic kidney injury in nephrolithiasis.Curr Opin Nephrol Hypertens.2014; 23:385-90.

[4] El Imam M, Omran M, Nugud F, Elsabiq M, Saad K, Taha O. Obstructive Uropathy in Sudanese Patients. Saudi J Kidney Dis Transpl.2006; 17:415-19.

[5] Daniele Bianchi, Giuseppe Vespasiani, and Pierluigi Bove .Acute kidney injury due to bilateral ureteral obstruction in children. World J Nephrol.2014; 3:182-92.

[6] Ibrahim AG, Aliyu S, Ali N. Bilateral pelvi-ureteric junction obstruction: our experience in a developing country. Niger J Clin Pract.2014; 17:267-69.

[7] Wang SJ, Mu XN, Zhang LY, Liu QY, Jin XB. The incidence and clinical features of acute kidney injury secondary to ureteral calculi.Urol Res.2012; 40:345-48.

[8] Chevalier RL, Klahr S. Therapeutic approaches in obstructive uropathy.SeminNephrol.1998; 18:652-58.

[9] Lucarelli G, Ditonno P, Bettocchi C, Grandaliano G, Gesualdo L, Selvaggi FP, Battaglia M. Delayed relief of ureteral obstruction is implicated in the long-term development of renal damage and arterial hypertension in patients with unilateral ureteral injury.J Urol.2013; 189:960-65.

[10] Nayyar R, Sarda AK, Kaza RCM, Anand VJ. The obstructed kidney. Indian J Surg.2005; 67:2128.

[11] Singh SM, Yaday R, Gupta NP, S.N.Wadhwa. The management of renal and ureteric calculi in renal failure. Br J Urol.1982; 54:455-57.

Citation: Dilip Kumar Pal and Surajit Sasmal.Clinical Profile and Treatment Outcome of Obstructive Uropathy in a Tertiary Care Centre. ARC Journal of Urology. 2017;2(1):5-10.

Copyright: @ 2017 Dilip Kumar Pal and Surajit Sasmal. This is an open-access article distributed under the terms of the Creative Commons Attribution License, which permits unrestricted use, distribution, and reproduction in any medium, provided the original author and source are credited. 